\title{
PERMUTATION POLYNOMIALS WITH EXPONENTS IN AN ARITHMETIC PROGRESSION
}

\author{
Young Ho PARK AND JUNE BOK LEE
}

\begin{abstract}
We examine the permutation properties of the polynomials of the type $h_{k, r, s}(x)=$ $x^{r}\left(1+x^{s}+\cdots+x^{s k}\right)$ over the finite field $\mathbb{F}_{q}$ of characteristic $p$. We give sufficient and necessary conditions in terms of $k$ and $r$ for $h_{k, r, 1}(x)$ to be a permutation polynomial over $\mathbb{F}_{q}$ for $q=p$ or $p^{2}$. We also prove that if $h_{k, r, s}(x)$ is a permutation polynomial over $\mathbb{F}_{q}$, then $(k+1)^{s}= \pm 1$.
\end{abstract}

\section{INTRODUCTION}

Let $\mathbb{F}_{q}$ be the finite field of $q=p^{n}$ elements of characteristic $p$. A polynomial $h(x) \in \mathbb{F}_{q}[x]$ is called a permutation polynomial (abbreviated to PP) over $\mathbb{F}_{q}$ if it induces a bijection on $\mathbb{F}_{q}$. In this article, we shall examine permutation properties of the polynomials

$$
h_{k, r, s}(x)=x^{r}\left(1+x^{s}+\cdots+x^{s k}\right)
$$

over $\mathbb{F}_{q}$, where $k, r, s$ are positive integers. These are the generalisations of the polynomials of the type $h_{k}(x)=1+x+\cdots+x^{k}$, whose permutation properties were completely characterised by Matthews when $q$ is odd [3]:

THEOREM A. For $q=p^{n}$ odd, $1+x+\cdots+x^{k}$ is a permutation polynomial over $\mathbb{F}_{q}$ if and only if $k \equiv 1(\bmod p(q-1))$.

Let

$$
d=\frac{q-1}{(s, q-1)}, \text { and } S=\left\{x \in \mathbb{F}_{q} \mid x^{s}=1\right\}
$$

There are two permuting classes as given in [3]. The proof of the following theorem is essentially the same as that given in [3], with a minor correction. We include it for the reader's convenience.

THEOREM B. $h_{k, r, s}(x)$ is a permutation polynomial over $\mathbb{F}_{q}$ if one of the following conditions holds:
(1) $k+1 \equiv 1(\bmod d), k+1 \in S$ and $(r, q-1)=1$;
(2) $k+1 \equiv-1(\bmod d),-(k+1) \in S$ and $(r-s, q-1)=1$.

Received 14th July, 1997

This work is supported by BSRI-96-1423 and by Yonsei Academic Research Grant, 1996.

Copyright Clearance Centre, Inc. Serial-fee code: 0004-9729/98 \$A2.00+0.00. 
Proof: Suppose (1). For any $a \in \mathbb{F}_{q}$, we have

$$
h_{k, r, s}(a)= \begin{cases}a^{r} \frac{1-a^{s(k+1)}}{1-a^{s}}=a^{r} & \text { if } a^{s} \neq 1, \\ (k+1) a^{r} & \text { if } a^{s}=1\end{cases}
$$

Since $k+1 \in S$ and $(r, q-1)=1$, we see that $(k+1) x^{r}$ maps $S$ onto $S$ and $x^{r}$ maps $\mathbb{F}_{q}-S$ onto inself.

Now suppose (2). Then

$$
h_{k, r, s}(a)= \begin{cases}a^{r} \frac{1-a^{s(k+1)}}{1-a^{s}}=-a^{r-s} & \text { if } a^{s} \neq 1, \\ (k+1) a^{r} & \text { if } a^{s}=1\end{cases}
$$

Since $-(k+1) \in S$ and $(r-s, q-1)=1$, we see that $(k+1) x^{r}$ maps $S$ onto $(-1)^{s} S$, and $-x^{r-s}$ maps $\mathbb{F}_{q}-S$ onto $\mathbb{F}_{q}-(-1)^{s} S$.

In his Ph.D dissertation Matthews has conjectured that the converse of Theorem B holds. We shall prove this conjecture for $q=p$ or $p^{2}$ (Theorem 4.6) and also prove that $\pm(k+1) \in S$ if $h_{k, r, s}(x)$ is a PP (Theorem 4.7). It is worth noting that, under the assumption that $h_{k, r, s}(x)$ is a permutation polynomial over $\mathbb{F}_{q}$, the conditions $k+1 \equiv 1(\bmod d), k+1 \in S$ force $(r, q-1)=1$ and the conditions $k+1 \equiv-1$ $(\bmod d),-(k+1) \in S$ imply $(r-s, q-1)=1$. As a consequence of Theorem 4.7, it remains to show that $k+1 \equiv \pm 1(\bmod d)$ to prove this conjecture.

The Hermite criterion will be used in the sequel [2];

THE Hermite CRITERIon. $f(x) \in \mathbb{F}_{q}[x]$ is a permutation polynomial over $\mathbb{F}_{q}$ if and only if the following conditions hold:

(1) $f$ has exactly one root in $\mathbb{F}_{q}$;

(2) for each integer $t$ with $1 \leqslant t \leqslant q-2$ and $t \not \equiv 0(\bmod p)$, the reduction of $f(x)^{t}\left(\bmod \left(x^{q}-x\right)\right)$ has degree $\leqslant q-2$.

\section{Preliminary Results.}

Clearly, $h_{k, r, s}(x)$ and $h_{k, r^{\prime}, s}(x)$ are equal as functions on $\mathbb{F}_{q}$ if $r \equiv r^{\prime}(\bmod q-1)$. For $k$, we have the following:

PROPOSITION 2.1. If $k \equiv l(\bmod p(q-1) /(s, q-1))$, then $h_{k, r, s}(a)=h_{l, r, s}(a)$ for all $a \in \mathbb{F}_{q}$.

PROOF: If $a^{s}=1$, then $h_{k, r, s}(a)=a^{r}(k+1)=a^{r}(l+1)=h_{l, r, s}(a)$. If $a^{s} \neq 1$, then

$$
h_{k, r, s}(a)=a^{r} \frac{a^{s(k+1)}-1}{a^{s}-1}=a^{r} \frac{a^{s(l+1)}-1}{a^{s}-1}=h_{l, r, s}(a)
$$


since $k \equiv l(\bmod (q-1) /(s, q-1))$ if and only if $s k \equiv s l(\bmod q-1)$.

This Proposition justifies the following notational convention. For negative integers $k, r, h_{k, r, s}(x)$ will mean $h_{k^{\prime}, r^{\prime}, s}(x)$, where $k^{\prime}, r^{\prime}$ are positive integers with $k^{\prime} \equiv k$ $(\bmod p(q-1) /(s, q-1))$ and $r^{\prime} \equiv r(\bmod q-1)$.

PROPOSITION 2.2. If $h_{k, r, s}(x)$ is a PP over $\mathbb{F}_{q}$, then

$$
\left(k+1, \frac{p(q-1)}{s, q-1}\right)=1 \text {. }
$$

Proof: Suppose $h_{k, r, s}(x)$ is a PP over $\mathbb{F}_{q}$. First, $h_{k, r, s}(1)=k+1 \neq h_{k, r, s}(0)=0$ $(\bmod p)$, that is, $(k+1, p)=1$. Since $h_{k, r, s}(a)=0$ if and only if $a^{r}=0$ or $1+a^{s}+\cdots+$ $a^{s k}=0$, there is no $a$ such that $a^{s} \neq 1$ and $1+a^{s}+\cdots+a^{s k}=\left(a^{s(k+1)}-1\right) /\left(a^{s}-1\right)=$ 0 . Thus if we let $N_{1}=\left\{a \mid a^{s}=1\right\}, N_{2}=\left\{a \mid a^{s(k+1)}=1\right\}$, then $N_{1}=N_{2}$. But $\left|N_{1}\right|=(s, q-1)=(s(k+1), q-1)=\left|N_{2}\right|$. Let $s=(s, q-1) s_{0}, q-1=$ $(s, q-1) q_{0}$ with $\left(s_{0}, q_{0}\right)=1$. Then $(s(k+1), q-1)=(s, q-1)\left((k+1) s_{0}, q_{0}\right)=$ $(s, q-1)\left(k+1, q_{0}\right)$. Hence $\left(k+1, q_{0}\right)=1$. Thus we have $1=\left(k+1, p q_{0}\right)=(k+$ $1, p(q-1) /(s, q-1))$.

Proposition 2.3. $h_{k, r, s}(x)$ is a PP if and only if $h_{-k-2, s-r, s}(x)$ is a PP.

Proof: We show that $h_{k, r, s}(a)=-h_{-k-2, s-r, s}\left(a^{q-2}\right)$ for all $a \in \mathbb{F}_{q}$. If $a^{s}=1$, then $h_{k, r, s}(a)=(k+1) a^{r}=-(-k-2+1)\left(a^{-1}\right)^{-r}\left(a^{-1}\right)^{s}=-h_{-k-2,-r+s, s}\left(a^{-1}\right)$. If $a^{s} \neq 0,1$, then

$$
-h_{-k-2,-r+s, s}\left(\frac{1}{a}\right)=-\left(\frac{1}{a}\right)^{(-r+s)} \frac{\left(\frac{1}{a}\right)^{s(-k-1)}-1}{\left(\frac{1}{a}\right)^{s}-1}=-a^{r} \frac{a^{s(k+1)}-1}{1-a^{s}}=h_{k, r, s}(a) .
$$

PRoposition 2.4. $h_{k, r, s}(x)$ is a PP over $\mathbb{F}_{q}$ if and only if $h_{k,-r-k s, s}(x)$ is a PP over $\mathbb{F}_{q}$.

Proof: We have $h_{k, r, s}\left(a^{q-2}\right)=h_{k,-r-k s, s}(a)$ for all $a \in \mathbb{F}_{q}$.

Let $(s, q-1)=s^{\prime}$. We can choose an integer $t$ relatively prime to $q-1$ such that $s t \equiv s^{\prime}(\bmod q-1)$. Since $x^{t}$ is a PP, $h_{k, r, s}(x)$ is a PP if and only if the composition $h_{k, r, s}\left(x^{t}\right)=h_{k, r t, s t}(x)$ is a PP. Now

$$
h_{k, r t, s t}(x) \equiv x^{r t}\left(1+x^{s^{\prime}}+\cdots+x^{s^{\prime} k}\right) \equiv h_{k, r t, g^{\prime}}(x) \quad\left(\bmod \left(x^{q}-x\right)\right)
$$

with $s^{\prime} \mid(q-1)$. Thus it suffices to consider the polynomials $h_{k, r, s}(x)$ with $s \mid(q-1)$. 
Now let $(r, s)=e$. If $(e, q-1) \neq 1$, then the equation $x^{e}=1$ has $(e, q-1)$ solutions and $h_{k, r, s}(x)$ sends all solutions of $x^{e}=1$ to $k+1$, so that $h_{k, r, s}(x)$ is not a PP. If $(e, q-1)=1$, then $x^{e}$ is a PP and $h_{k, r, s}(x)=h_{k,(r / e),(s / e)}\left(x^{e}\right)$ is a PP if and only if $h_{k,(r / e),(s / e)}(x)$ is a PP.

In conclusion, it is enough to consider the cases that $(r, s)=1$ and $s \mid(q-1)$. From now on, we shall always assume that

$$
(r, s)=1 \text { and } s \mid(q-1)
$$

so that

$$
d=\frac{q-1}{s}
$$

\section{Circulant matrices.}

We review elementary facts about circulant matrices. A circulant matrix of order $n$ is an $n \times n$ matrix of the form

$$
\operatorname{circ}\left(c_{0}, c_{1}, \ldots, c_{n-1}\right)=\left(\begin{array}{cccc}
c_{0} & c_{1} & \ldots & c_{n-1} \\
c_{n-1} & c_{0} & \ldots & c_{n-2} \\
\vdots & \vdots & & \vdots \\
c_{1} & c_{2} & \ldots & c_{0}
\end{array}\right)
$$

For a polynomial $f(x)=c_{0}+c_{1} x+c_{2} x^{2}+\cdots+c_{n-1} x^{n-1}, C_{f}=\operatorname{circ}\left(c_{0}, c_{1}, \ldots, c_{n-1}\right)$ is called the circulant matrix of $f$. It is well known that if a field $F$ has a primitive $n$th root of unity $\zeta$ and $f(x)=c_{0}+c_{1} x+\cdots+c_{n-1} x^{n-1} \in F[x]$, then $C_{f}$ can be put into a diagonalised form as follows $[1,4]$;

$$
C_{f} \sim\left(\begin{array}{ccccc}
f(1) & & & & \\
& f(\zeta) & & & \\
& & f\left(\zeta^{2}\right) & & \\
& & & \ddots & \\
& & & & f\left(\zeta^{n-1}\right)
\end{array}\right)
$$

Suppose $f(x)=a_{1} x+\cdots+a_{q-1} x^{q-1} \in \mathbb{F}_{q}$ is a PP over $\mathbb{F}_{q}$. The Hermite criterion implies $a_{q-1}=0$. Considering the circulant matrix $M_{f}=\operatorname{circ}\left(0, a_{1}, a_{2}, \ldots, a_{q-2}\right)$ of order $q-1$, we then have

$$
\operatorname{det} M_{f}=\prod_{a \in \mathbb{F}_{q}^{*}} f(a)=\prod_{a \in \mathbb{F}_{q}^{*}} a=-1
$$

For $a \in \mathbb{F}_{q}$ and positive integer $m$, we denote by $a_{(m)}$ the row vector $(a, a, \cdots, a)$ with $m a$ 's. 
Let $C=\operatorname{circ}\left(a_{(m)}, b_{(n-m)}\right)$ be an $n \times n$ circulant matrix with $m a$ 's and $(n-m)$ $b$ 's, where $a \neq b$. Then, using (3.1), it is not difficult to show

$$
\operatorname{det} C= \begin{cases}(m a+(n-m) b)(a-b)^{n-1} & \text { if }(m, n)=1 \\ 0 & \text { otherwise. }\end{cases}
$$

It is also clear that

$$
\operatorname{det} \operatorname{circ}\left(a_{0}, a_{1}, a_{2}, \ldots, a_{n-1}\right)=(-1)^{n-1} \operatorname{det} \operatorname{circ}\left(a_{1}, a_{2}, \ldots, a_{n-1}, a_{1}\right) .
$$

\section{Main Results}

Returing to PP's, we first consider the case $s=1$ and write $h_{k, r}(x)$ for $h_{k, r, 1}(x)$. Assume $h_{k, r}(x)$ is a PP, and write

$$
r+k=l(q-1)+m, \text { where } 0 \leqslant m<q-1 .
$$

Let $f(x)=h_{k, r}(x)\left(\bmod \left(x^{q}-x\right)\right)$ with $\operatorname{deg}(f)<q$. Then

$$
f(x)=\left\{\begin{array}{cc}
l\left(x+\cdots+x^{r-1}\right)+(l+1)\left(x^{r}+\cdots+x^{m}\right) & \\
+l\left(x^{m+1}+\cdots+x^{q-1}\right), & \text { if } m \geqslant r \\
l\left(x+\cdots+x^{m}\right)+(l-1)\left(x^{m+1}+\cdots+x^{r-1}\right) & \\
+l\left(x^{r}+\cdots+x^{q-1}\right), & \text { if } m<r .
\end{array}\right.
$$

By the Hermite criterion, $l \equiv 0(\bmod p)$, and hence

$$
f(x)= \begin{cases}x^{r}+\cdots+x^{m}, & \text { if } m \geqslant r \\ -x^{m+1}-\cdots-x^{r-1}, & \text { if } m<r .\end{cases}
$$

First consider the case $m \geqslant r$, and let

$$
M_{f}=\operatorname{circ}\left(0_{(r)}, 1_{(m-r+1)}, 0_{(q-2-m)}\right)
$$

be the circulant matrix of $f(x)$ of order $(q-1) \times(q-1)$. Since

$$
m-r+1 \equiv k+1 \quad(\bmod p(q-1))
$$

we have $(m-r+1, q-1)=1$ by Propostion 2.2. Hence, by (3.3) and (3.4),

$$
\operatorname{det} M_{f}=(-1)^{r(q-2)} \operatorname{det} \operatorname{circ}\left(1_{(m-r+1)}, 0_{(q-2-m+r)}\right)=(-1)^{r(q-2)}(m-r+1) .
$$

By (3.2) and (4.1) we thus have

(4.2) $k+1 \equiv m-r+1 \equiv(-1)^{r(q-2)} \operatorname{det} M_{f} \equiv(-1)^{r(q-2)-1} \equiv(-1)^{r-1} \quad(\bmod p)$.

Similar argument shows that (4.2) holds also when $m<r$. Thus we have proved: 
THEOREM 4.1. If $x^{r}\left(1+x+\cdots+x^{k}\right)$ is a PP over $\mathbb{F}_{q}$, then

$$
k+1 \equiv(-1)^{r-1} \quad(\bmod p) \text {. }
$$

Now we prove that the conjecture is true for $s=1$ and $q=p$.

ThEOREM 4.2. $h_{k, r}(x)=x^{r}\left(1+x+\cdots+x^{k}\right)$ is a PP over $\mathbb{F}_{p}$ if and only if one of the following conditions holds:

(1) $k+1 \equiv 1(\bmod p(p-1))$ and $(r, p-1)=1$;

(2) $k+1 \equiv-1(\bmod p(p-1))$ and $(r-1, p-1)=1$.

ProOf: The claim is easy for $p=2$, so we assume that $p$ is odd. By Proposition 2.1 , we may assume that $1 \leqslant k \leqslant p(p-1)$. We may also assume that $1 \leqslant r \leqslant p-1$. As above, write

$$
r+k=l(p-1)+m, \text { with } 0 \leqslant m \leqslant p-2 .
$$

We know that $l \equiv 0(\bmod p)$ and $m-r+1 \equiv \pm 1(\bmod p)$. Since $-(p-2) \leqslant m-r+1 \leqslant$ $p$, we must have $m-r+1=1,-1$ or $p-1$.

Case 1. $m-r+1=1$ : Then $k=l(p-1)=p(p-1)$ and $h_{k, r}(a)=$ $a^{r}\left(1+a+\cdots+a^{p(p-1)}\right)=a^{r}$ for all $a \in \mathbb{F}_{p}$. In this case, $h_{k, r}(x)$ is a PP over $\mathbb{F}_{p}$ if and only if $(r, q-1)=1$.

Case 2. $\quad m-r+1=-1$ : Then $k=l(p-1)-2=p(p-1)-2$, and $h_{k, r}(a)=-a^{r-1}$ for all $a \in \mathbb{F}_{p}$. So $h_{k, r}(x)$ is a PP if and only if $(r-1, q-1)=1$.

Case 3. $m-r+1=p-1$ : Then $k=l(p-1)+p-2=p-2$ and, for $a \neq 0,1$, we have $h_{k, r}(a)=a^{r}\left(1+a+\cdots+a^{p-2}\right)=a^{r}\left(a^{p-1}-1\right) /(a-1)=0$. Thus $h_{k, r}(x)$ is not a PP over $\mathbb{F}_{p}$.

Before we proceed to the case $q=p^{2}$, we need several observations.

LEMMA 4.3. Suppose $r<q$ and $k \leqslant p(q-1)$. If $h_{k, r}(x)=x^{r}\left(1+x+\cdots+x^{k}\right)$ is a $P P$ over $\mathbb{F}_{q}$, then $r+k<q-1$ or $p(q-1) \leqslant r+k<(p+1)(q-1)$.

Proof: The coefficient of $x^{q-1}$ of $h_{k, r}(x)\left(\bmod x^{q}-x\right)$ is $[(r+k) /(q-1)]$. Hence, by the Hermite criterion, $[(r+k) /(q-1)] \equiv 0(\bmod p)$. Since $r+k \leqslant$ $q-1+p(q-1)$, we have $[(r+k) /(q-1)]=0$ or $p$.

LEMMA 4.4. Let $r<q, q$ odd, and $k \leqslant p(q-1)$. If $(q-1) / 2 \leqslant r+k<q-1$, and if $h_{k, r}(x)$ is a $P P$ over $\mathbb{F}_{q}$, then $r \equiv 0(\bmod p)$ or $r+k+1 \equiv 0(\bmod p)$.

ProOF: We have

$$
h_{k, r}(x)^{2}=x^{2 r}\left(1+2 x+3 x^{2}+\cdots+(k+1) x^{k}+k x^{k+1}+(k-1) x^{k+2}+\cdots+x^{2 k}\right)
$$

Hence, the coefficient of $x^{q-1}$ in $h_{k, r}(x)^{2}$ is given by $(2 k+1)-(q-1-2 r)=2 k+$ $2 r+2-q$ if $2 r+k<q-1$, or given by $(q-1-2 r)+1=q-2 r$ if $2 r+k \geqslant q-1$. By the Hermite criterion, the result follows. 
Proposition 4.5. Suppose $q$ is odd and $1 \leqslant k \leqslant p(q-1)$. If $h_{k, r}(x)$ is a PP over $\mathbb{F}_{q}$, then

$$
k+1=t p(p-1) \pm 1
$$

for some integer $t$ such that $1 \leqslant t \leqslant(q-p) /(p(p-1))$ or $(q / p) \leqslant t \leqslant(q-1) /(p-1)$.

Proof: We may assume that $1 \leqslant r \leqslant q-1$. By Lemma 4.3, we then have $1 \leqslant k<q-1$ or $(p-1)(q-1) \leqslant k \leqslant p(q-1)$. Since $k=t p(p-1)$ or $k=t p(p-1)-2$ for some $t$ by Theorem 4.2 , we have $1 \leqslant t \leqslant(q-1) /(p(p-1))+(\delta / p(p-1))$ or $(q / p)-(1 / p)+(\delta / p(p-1)) \leqslant t \leqslant(q-1) /(p-1)+(\delta / p(p-1))$, where $\delta=0,2$. Note that $(q-1) /(p(p-1))+(\delta / p(p-1))=(q / p-1) /(p-1)+(p-1+\delta) /(p(p-1))$, and $(q / p-1) /(p-1)$ is an integer. When $q$ is odd, we have $(p-1+\delta) /(p(p-1))<1$, $-(1 / p)+(\delta / p(p-1))>-1$ and $(\delta / p(p-1))<1$ for $\delta=0,2$, and thus the claim follows.

THEOREM 4.6. Let $q=p$ or $q=p^{2}$. Then $h_{k, r}(x)=x^{r}\left(1+x+\cdots+x^{k}\right)$ is a $P P$ over $\mathbb{F}_{q}$ if and only if one of the following conditions holds:

(1) $k+1 \equiv 1(\bmod p(q-1))$ and $(r, q-1)=1$;

(2) $k+1 \equiv-1(\bmod p(q-1))$ and $(r-1, q-1)=1$.

Proof: Suppose $h_{k, r}(x)$ is a PP over $\mathbb{F}_{q}$. We may assume that $r<q$ and $k \leqslant p(q-1)$. Let $q=p^{2}$.

First we consider the case $q=4$. By Theorem $4.1, k=2,4$ or $6 . \mathbb{F}_{4}=\{0,1, \alpha$, $1+\alpha\}$ where $\alpha^{2}=\alpha+1$. If $k=2, h_{k, r}(\alpha)=\alpha^{r}\left(1+\alpha+\alpha^{2}\right)=0$, and so $h_{k, r}(x)$ is not a PP over $\mathbb{F}_{q}$. If $k=4$, then $h_{k, r}(a)=-a^{r-1}$ for $a \neq 1$ by (1.2) and thus $h_{k, r}(x)$ is a PP over $\mathbb{F}_{q}$ if and only if $(r-1, q-1)=1$ (Case (1)). If $k=6$, then $h_{k, r}(a)=a^{r}$ for $a \neq 0$ by (1.1) and then $h_{k, r}(x)$ is a PP over $\mathbb{F}_{q}$ if and only if $(r, q-1)=1$ (Case $(2))$.

Now consider for odd $q=p^{2}$. By Proposition $4.5, k+1=t p(p-1) \pm 1$ for some $t$ such that $1 \leqslant t \leqslant 1$ or $p \leqslant t \leqslant p+1$. So the possible values of $t$ are $1, p$ or $p+1$. We shall show that $t \neq 1, p$

First, assume $t=1$ so that $k=p(p-1)$ or $k=p(p-1)-2$. If $r<p-1$, then

$$
q-1>r+k>k \geqslant p^{2}-p-2 \geqslant \frac{q-1}{2} .
$$

Lemma 4.4 implies that $r \equiv 0(\bmod p)$ or $r \pm 1 \equiv 0(\bmod p)$. This is impossible since $r<p-1$. Thus $r \geqslant p-1$. If $k=p(p-1)-2$ and $r=p-1$, then $q-1\rangle$ $r+k=q-3>(q-1) / 2$. Again Lemma 4.4 implies $r=p-1 \equiv 0(\bmod p)$ or $r+k+1=q-2 \equiv 0(\bmod p)$, which is absurd. If $k=p(p-1)-2$ and $r=p$, then $(r-1, p-1)=p-1 \neq 1$, and thus $h_{k, r}(x)$ is not a PP by Theorem 4.2. Finally, if $p-1 \leqslant r \leqslant q-1$ with $k=p(p-1)$ or $p+1 \leqslant r \leqslant q-1$ with $k=p(p-1)-2$, 
then $q-1 \leqslant r+k \leqslant 2(q-1)$. So the coefficient of $x^{q-1}$ in $h_{k, r}(x)\left(\bmod \left(x^{q}-x\right)\right)$ is nonzero. By the Hermite criterion, $h_{k, r}(x)$ is not a PP over $\mathbb{F}_{q}$.

Now consider the case $t=p$. Then $k=p^{2}(p-1)$ or $k=p^{2}(p-1)-2$, so that $p(q-1)-k-2$ is $p(p-1)-2$ or $p(p-1)$, respectively. Recall that $h_{k, r}(x)$ is a PP if and only if $h_{p(q-1)-k-2, q-r}(x)$ is a PP (Proposition 2.3). Thus this case reduces to the case $t=1$ and hence $h_{k, r}(x)$ is not a PP.

Therefore $t=p+1$ and $k+1=p(q-1) \pm 1$. The remaining assertions are now clear by (1.1) and (1.2).

The tensor product or Kronecker product $A \otimes B$ of two matrices $A, B$ is defined by

$$
A \otimes B=\left(\begin{array}{cccc}
b_{11} A & b_{12} A & \ldots & b_{1 \nu} A \\
b_{21} A & b_{22} A & \ldots & b_{2 \nu} A \\
\vdots & \vdots & & \vdots \\
b_{\mu 1} A & b_{\mu 2} A & \ldots & b_{\mu \nu} A
\end{array}\right)
$$

where $B=\left(b_{i j}\right)$ is a $\mu \times \nu$ matrix. It is well known that

$$
\operatorname{det} A \otimes B=(\operatorname{det} A)^{\nu}(\operatorname{det} B)^{\mu}
$$

if $A$ is a $\mu \times \mu$ matrix, and $B$ is a $\nu \times \nu$ matrix [4].

Towards the conjecture, we consider the general $s \mid(q-1)$.

THEOREM 4.7 . If $h_{k, r, s}(x)$ is a PP over $\mathbb{F}_{q}$, then

$$
(k+1)^{s} \equiv(-1)^{r-1} \quad(\bmod p) .
$$

Furthermore,

$$
k+1 \in S \text { or }-(k+1) \in S .
$$

Proof: Let $s \neq 1$. Write $r+k s=l(q-1)+m, 0 \leqslant m<q-1$ as before, and let $r=l_{0} s+r_{0}$ with $0<r_{0}<s$. Let $f(x)=h_{k, r, s}(x)\left(\bmod \left(x^{q}-x\right)\right)$ with $\operatorname{deg}(f)<q$. If $m \geqslant r$, then

$$
\begin{aligned}
f(x)=l\left(x^{r_{0}}+x^{r_{0}+s}+\cdots+x^{r-s}\right)+(l+1) & \left(x^{r}+x^{r+s}+\cdots+x^{m}\right) \\
& +l\left(x^{m+s}+x^{m+2 s}+\cdots+x^{q-1+r_{0}-s}\right),
\end{aligned}
$$

and if $m<r$, then

$$
\begin{aligned}
f(x)=l\left(x^{r_{0}}+x^{r_{0}+s}+\cdots+x^{m}\right)+(l-1)\left(x^{m+s}\right. & \left.+x^{m+2 s}+\cdots+x^{r-s}\right) \\
& +l\left(x^{r}+x^{r+s}+\cdots+x^{q-1+r_{0}-s}\right) .
\end{aligned}
$$


As before, let $M_{f}$ be the circulant matrix of order $(q-1) \times(q-1)$ with the first row vector $\left(0, a_{1}, a_{2}, \ldots, a_{q-2}\right)$ where $f(x)=a_{1} x+a_{2} x^{2}+\cdots+a_{q-2} x^{q-2}$.

First, consider the case $m \geqslant r$. We have

$$
\begin{aligned}
\operatorname{det} M_{f}= & \operatorname{det} \operatorname{circ}\left(0_{\left(r_{0}\right)}, l, 0_{(s-1)}, \ldots, l, 0_{(s-1)}, l+1,0_{(s-1)}, \ldots, l+1,0_{(s-1)},\right. \\
& \left.l, 0_{(s-1)}, \ldots, l, 0_{\left(s-1+s-r_{0}\right)}\right) \\
= & (-1)^{r_{0}(q-2)} \operatorname{det} \operatorname{circ}\left(l, 0_{(s-1)}, \ldots, l, 0_{(s-1)}, l+1,0_{(s-1)}, \ldots, l+1,0_{(s-1)},\right. \\
& \left.l, 0_{(s-1)}, \ldots, l, 0_{(s-1)}\right) \\
= & (-1)^{r_{0}(q-2)} \operatorname{det}\left(I_{s} \otimes \operatorname{circ}(\overbrace{l, l, \ldots, l, \overbrace{l+1, \ldots, l+1}^{\left(r-r_{0}\right) / s}, \overbrace{l, \ldots, l}^{((m-r) / s)+1}))}^{d-\left(\left(m-r_{0}\right) / s\right)-1})\right. \\
= & (-1)^{r_{0}(q-2)}\left[\operatorname{det} \operatorname{circ}(\overbrace{l, l, \ldots, l, \overbrace{l+1, \ldots, l+1}^{\left(r-r_{0}\right) / s}, \overbrace{l, \ldots, l}^{((m-r) / s)+1})]^{d-\left(\left(m-r_{0}\right) / s\right)-1},}^{s},\right.
\end{aligned}
$$

where $I_{s}$ is the $s \times s$ identity matrix, and $d=(q-1) / s$. By (3.3) and (3.4),

$$
\begin{aligned}
& \operatorname{det} \operatorname{circ}(\overbrace{l, l, \ldots, l, \overbrace{l+1, \ldots, l+1}^{\left(r-r_{0}\right) / s}, \overbrace{l, \ldots, l}^{((m-r) / s)+1})}^{d-\left(\left(m-r_{0}\right) / s\right)-1}) \\
= & (-1)^{\left(r-r_{0}\right)(d-1) / s} \operatorname{det} \operatorname{circ}(\overbrace{l+1, \ldots, l+1}^{((m-r) / s)+1}, \overbrace{l, \ldots, l}^{d-((m-r) / s)-1}) \\
= & (-1)^{\left(r-r_{0}\right)(d-1) / s}\left(d l+\frac{m-r}{s}+1\right)=(-1)^{\left(r-r_{0}\right)(d-1) / s}(k+1) .
\end{aligned}
$$

But, for odd $q$, we have

$$
r_{0}(q-2)+\left[\frac{r-r_{0}}{s}(d-1)\right] s \equiv r_{0}+l_{0} s(d-1) \equiv r_{0}-l_{0} s \equiv r+l_{0} s=r \quad(\bmod 2) .
$$

Consequently, $(k+1)^{s}=(-1)^{r-1}$ by (3.2).

By a similar argument when $m<r$ we obtain

$$
\operatorname{det} M_{f}=(-1)^{r_{0}(q-2)+\left(m-r_{0}+2 s\right)(d-1)}(k+1)^{s} .
$$

For odd $q$, a short calculation shows that $r_{0}(q-2)+\left(m-r_{0}+2 s\right)(d-1) \equiv m \equiv$ $r+s k \equiv r(\bmod 2)$. Here, the last congruence follows beause if $s$ is odd, then $d$ is even and then $k$ is even by Proposition 2.2. Thus we always have $(k+1)^{s}=(-1)^{r-1}$.

Finally the last assertion of our Theorem is clear for even $q$. Assume $q$ is odd. If $r$ is odd, then $(k+1)^{s}=1$ so that $k+1 \in S$. On the other hand, if $r$ is even then $s$ must be odd, because if $r$ and $s$ were both even, then $h_{k, r, s}(x)$ would be a polynomial in $x^{2}$ and then $h_{k, r, s}(x)$ could not be a PP. Thus if $r$ is even, $(-(k+1))^{s}=1$. 


\section{REFERENCES}

[1] W.-S. Chou, Permutation polynomials over finite dields and combinatorial applications, (Ph.D. Dissertation) (The Pennsylvania State University, 1990).

[2] R. Lidl and H. Niederreiter, Finite fields, Encyclopedia Math. Appl., 20 (Addison-Wesley, Reading, MA, 1983).

[3] R. Matthews, 'Permutation properties of the polynomials $1+x+\cdots+x^{k}$ over a finite field', Proc. Amer. Math. Soc. 120 (1994), 47-51.

[4] M. Marvin and H. Minc, A survey of matrix theory and matrix inequalities (Dover, New York, 1964).

Department of Mathematics Kangwon National University

Chuncheon 200-701

Korea
Department of Mathematics

Yonsei University

Seoul 120-749

Korea 